\title{
NUMERICAL AND EXPERIMENTAL DETERMINATION OF WAVY FIN- TUBE SHAPE FACTOR
}

\author{
Yau Kar Hing ${ }^{1, *}$, W.M. Chin ${ }^{1}$ and M.R. Heikal ${ }^{2}$ \\ ${ }^{1}$ O.Y.L Research and Development Center \\ 47000 Sg. Buloh, Selangor, Malaysia. \\ Tel: +603 61570846; Fax: +603 61570869 . \\ ${ }^{2}$ Universiti Teknologi PETRONAS, \\ Bandar Seri Iskandar, 31750 Tronoh, Perak, Malaysia. \\ Tel: +605 368 8000; Fax: +605365 4075 . \\ *Email:kjljeff@gmail.com
}

\begin{abstract}
This paper presents the numerical and experimental investigations of a wavy fin-tube heat exchanger aimed at correctly accounting for all factors influencing the thermal performance of the exchanger. The shape factor for the complex heat conduction path in the wavy fin is determined by using computational analysis and validated experimentally by utilizing electrical analogy to obtain the electric resistance across the fin. This is used to back-calculate the conduction shape factor. In the experimental study, the potential difference, $V$ and current, $I$, was measured using a high precision data acquisition unit. The results were used to calculate the shape resistance which was compared with that obtained from the numerical model. Grid independence tests were performed on the model and several analytically derived standard shape factor formulae were also used for comparison with the model outputs. The deviation of the numerical results from the analytical formulae for the cases studied was less than $+1.2 \%$. The agreement between the experiments and the numerical model was within $+3.5 \%$. The results demonstrated the adequacy of the numerical approach to modeling the wavy fintube heat transfer. Effects such as differences in fin shape, fin length and waviness of the fin design on the shape factor were determined and discussed.
\end{abstract}

Keywords: Heat transfer; fin shape factor; electrical analogy.

\section{INTRODUCTION}

In air-cooled refrigerant condensers and evaporators, heat passes from the refrigerant to the air through a number of thermal resistance paths. The greatest complexity in analysis of these corresponds to conduction in air side of the fin due to its complex shape and waviness. Heat conduction in 2-dimensions involves the shape factor, $S$, that offers convenience in analyzing complex thermal systems. Shape factors for simple shapes such as plates, spheres, and hollow cylinders, are readily available from textbooks (Cengel, Boles, \& Kanoglu, 2007; Incropera \& De Witt, 2002). However, to handle complex systems bounded by two isothermal surfaces such as an elliptic cylinder or oblate sphere, the problem is much more difficult (Al- Doori, 2011; Fyrillas, 2008, 2010; Yovanovich, 1973). These complex shapes were studied as early as 1973 and only limited to shapes that were predefined in their studies. For complex 3-dimensional conduction problems, the analytical solution to obtaining shape factors requires a tedious process of the integration of formulae in the three orthogonal directions, as in a 
coordinate system such as the Cartesian system. An inappropriate approach to the coordinate system would lead to erroneous solutions. A complex shape is even more complex when it is solved with a transient case (Bart \& Hanjalić, 2003). Recently, with the advance of many numerical computational software packages, the solution to the shape factor for complex geometries has become more easily accessible. Study of 3dimensional enclosure for conduction shape factors is found to be scarce (Ishak, Tahseen, \& Rahman, 2013; Shrivastava \& Roemer, 2003, 2005; Syam Sundar \& Sharma, 2011; Teertstra, Yovanovich, \& Culham, 2005). In Teertstra et al. (2005), an analytical investigation of a rectangular shape with parallel pipe was performed and compared with a simulation using computational fluid dynamic software. Their model (Teertstra et al., 2005) was shown to be accurate within 5\%rms.

In this work, a novel method of obtaining the shape factor for a wavy fin-tube geometry by using field experiment and computational analysis is explained. The aim of this work was achieved by obtaining the shape factor of a wavy fin from a fin-tube heat exchanger. The shape factor was needed to calculate the resistance of the fin conduction for the total thermal resistance network of a fin-tube heat exchanger. The wavy fin used in the fin-tube heat exchanger, as illustrated in Figure 1, is a thin foil of aluminum pressed into wavy patterns and then punched with holes to fit with copper tubes. The punched holes are extruded with J-footed collars. The heat conduction path across the fin therefore consists of a wavy pattern of the fin foil and a J-footed collar. The experiment to obtain the shape factor is explained in the following section. The commercial software ANSYS was used to obtain the numerical results.
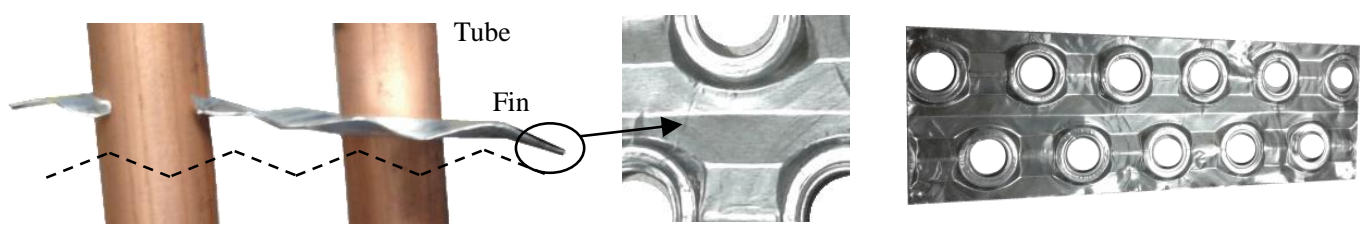

Figure 1. Illustration of an aluminum wavy fin

\section{METHODOLOGY}

\section{Experimental study}

Since electrical conduction and thermal conduction are analogous, conduction shape factor obtained by electrical measurements is identical to the thermal conduction shape factor. This principle of analogy is used in the field experiments performed on the fin. A flat constantan foil was used instead of the aluminum fin as its electrical resistivity, $\rho$, is one magnitude higher at $5.00 \times 10^{-7} \Omega . \mathrm{m}$ compared to $2.82 \times 10^{-8} \Omega . \mathrm{m}$ for the aluminum fin. The constantan foil was cut to resemble the shape of the aluminum fin used in the heat exchanger as shown in Figure 2. The aluminum fin specification follows the $9.52 \mathrm{~mm}$ fin-tube heat exchanger as set out in Table 1. 


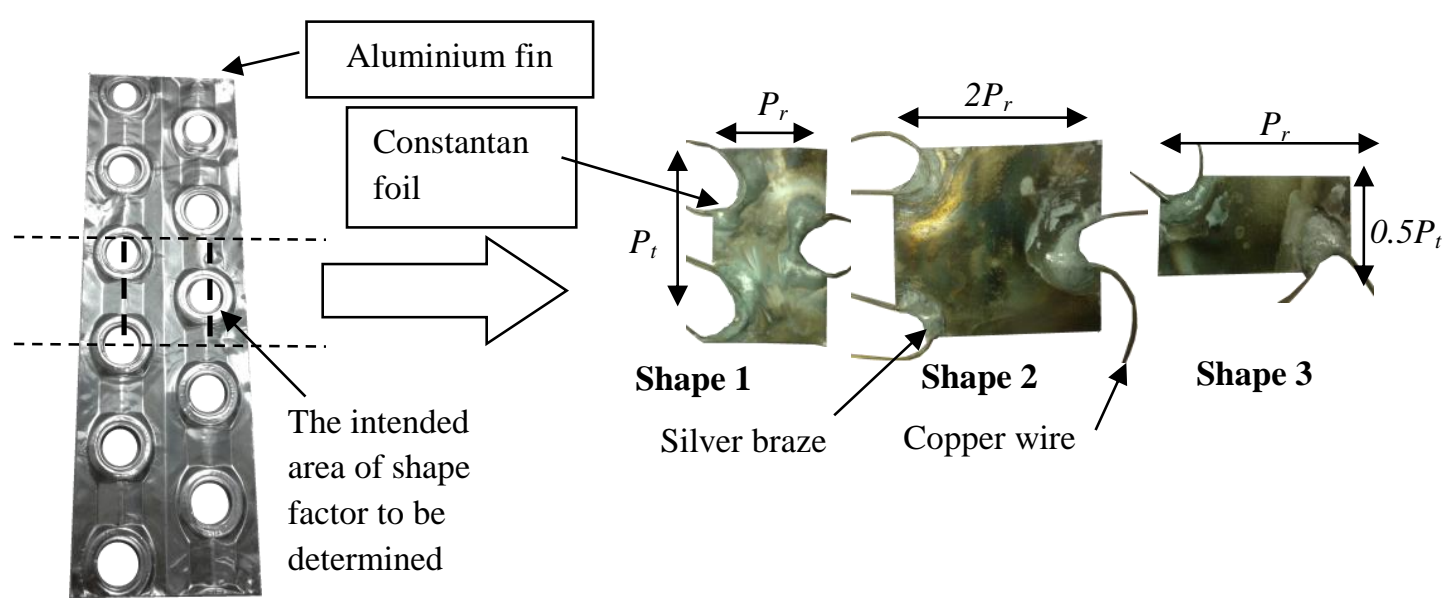

Figure 2. Illustration of the constantan shape for aluminum fin.

Table 1. Specifications of the aluminum fin and constantan foil.

\begin{tabular}{lc}
\hline Geometry & Specification \\
\hline Tube pitch, $P_{t}(\mathrm{~mm})$ & 22.0 \\
Row pitch, $P_{r}(\mathrm{~mm})$ & 25.4 \\
Tube diameter, $\varnothing(\mathrm{mm})$ & 9.52 \\
Aluminum fin thickness, $F_{t, a l}(\mathrm{~mm})$ & 0.11 \\
Constantan foil thickness, $F_{t, c t}(\mathrm{~mm})$ & 0.06 \\
\hline
\end{tabular}

Three samples with different shapes were cut and affixed with copper lead wires, as illustrated in Figure 2, by brazing using silver-aluminum flux. Two samples were prepared for each shape; thus a total of 6 samples were used in this experiment. For convenience, the constantan foils are named Shape 1, Shape 2 and Shape 3 for original size, larger size (dimensions increased by a factor of 2 maintaining geometric similarity) and smaller size (tube pitch reduced by half), respectively. Different sizes of constantan foil were used to allow more data for comparison and to determine how the shape factor would change accordingly. Methods of connecting the copper wires to the constantan foil were also investigated in this work. The first method used silver conductive paint (Madhusudana, 1996), which has characteristics such as adhesion, which is commonly used in the electrical industry to repair printed circuit boards. Silver conductive paint has a resistivity of $1.0 \times 10^{-3} \Omega$.m and electrical conductivity of $1.0 \mathrm{~S} / \mathrm{m}$. A second method involved brazing with particular fluxes such as silver flux (ULTRA FLUX) and cesiumaluminum flux (Al-Flux $028 \mathrm{Cs}$ ). The two fluxes were used to determine the effectiveness of bonding between the wires and the constantan foil. The fluxes were in the form of a paste. The composition of the silver flux was $40 \%$ potassium tetraborate, with the remainder containing other compounds, and the silver-aluminum flux consisted of approximately $22 \%$ aluminum and the remainder was a mixture of $\mathrm{Zn}-\mathrm{Si}$ and $\mathrm{Cs}$. When the copper wires were securely attached to the collars of the constantan foil, current was supplied to the fin via a direct current (D.C.) power pack, and the current rating, $I$ was measured with a D.C. micro-ammeter to ensure that the current was constant throughout the test. The potential difference, $V$, across the fin, was then measured with another D.C. micro-voltmeter. The schematic of the electrical circuit is shown in Figure 3. 


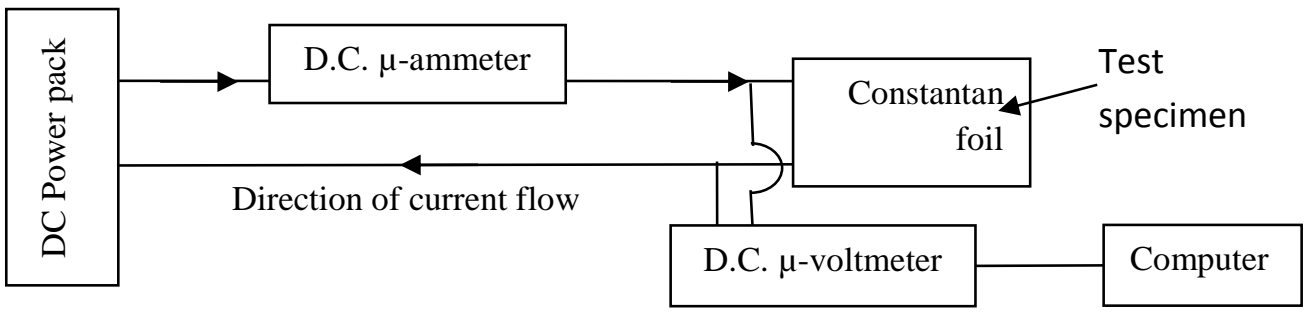

Figure 3. Schematic of the electrical circuit.

The test was performed by setting the current as $1.0 \mathrm{~A}, 1.5 \mathrm{~A}$ or $2.0 \mathrm{~A}$. The data, such as the potential difference, $V$, was captured through a computer using data acquisition software LabView. Prior to the logging the data, the experiment was allowed to stabilize for approximately 5 minutes. For each sample, the data was collected for a minute's duration with one second intervals between each point, and so approximately 60 data points were collected.

\section{Modeling of the Constantan Foil Sample}

The numerical model of the fin shape factor was solved using ANSYS (Version 14) with an electric analysis module built into the software. Three shapes were modelled following the samples for the field experiments as shown in Figure 4, and an additional 2 different shapes were modelled based on the actual shape used in the fin-and-tube heat exchanger shown in Figure 5.
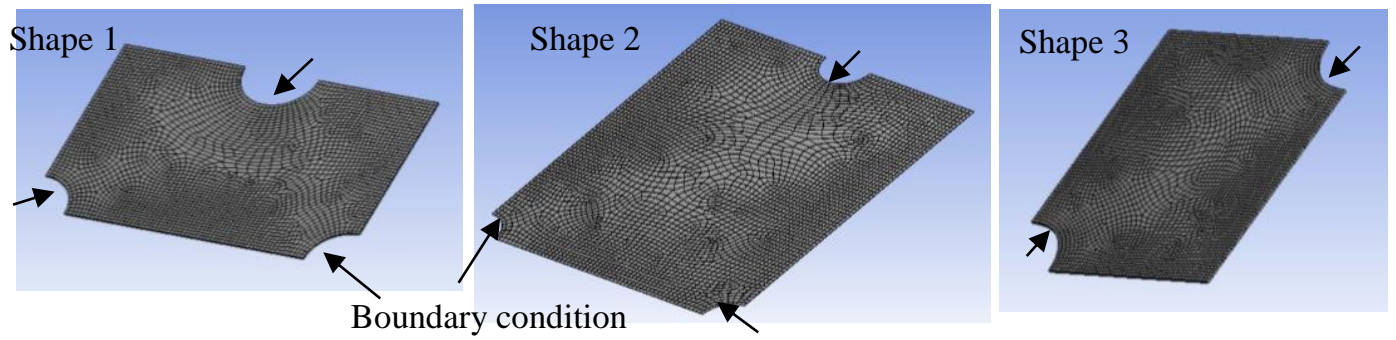

Figure 4. The validation models for numerical analysis.
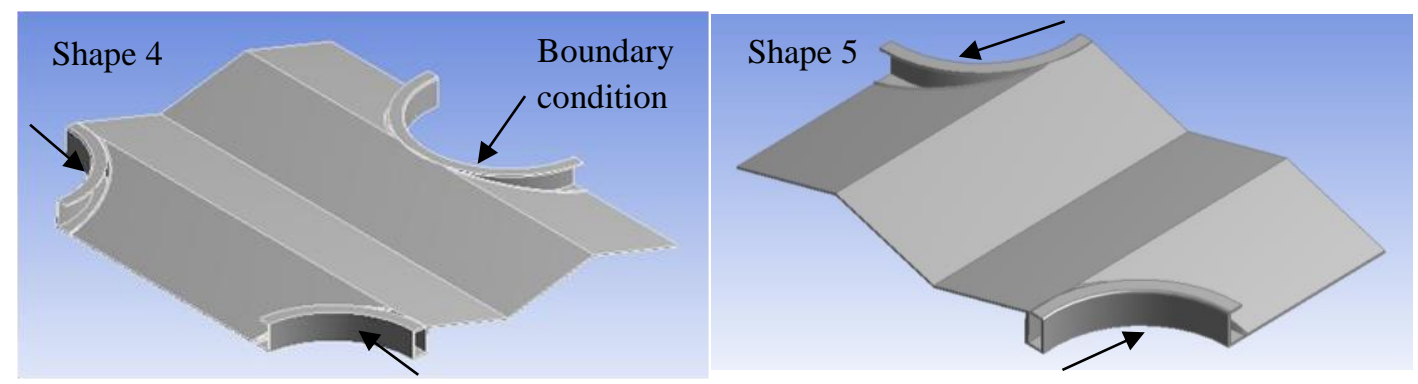

Figure 5. Wavy fin models for numerical analysis.

A total of 5 different shapes were used in the numerical analysis and for convenience, the models are labelled as Shapes 1, 2, 3, 4 and 5. Shapes 1 to 3 were for validation purposes, and Shapes 4 and 5 were to obtain the wavy-fin shape factor based on the numerical solution. 


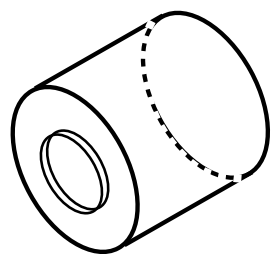

Round shape Area, $A_{1}$

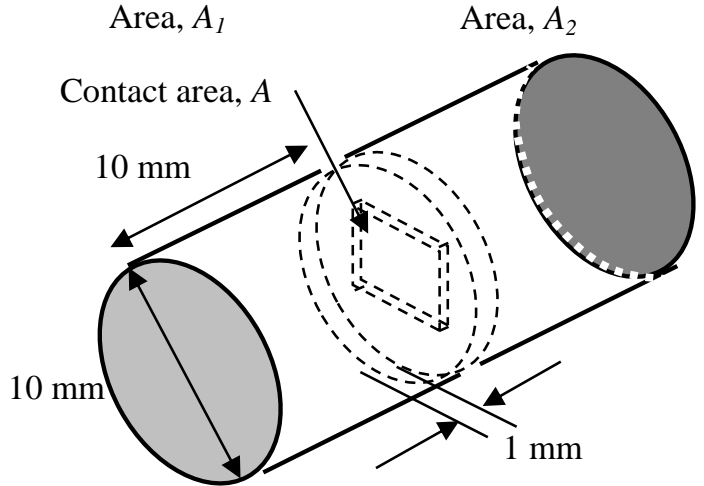

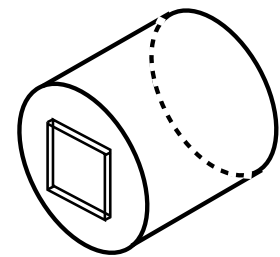

Square shape Square shap

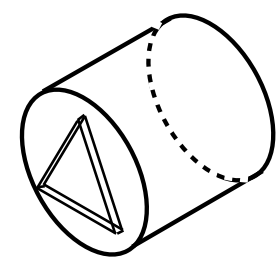

Triangle shape

Area, $A_{3}$

$A_{l}=$ Round

$A_{2}=$ Square

$A_{3}=$ Triangle

$A_{1}=A_{2}=A_{3}=19.64 \mathrm{~mm}^{2}$

Figure 6. Effect of shape on the thermal shape factor using the Holm tube model.

In addition, the thermal shape factor was compared with the electrical shape factor numerically by varying simple geometries such as triangle, circle and square, as shown in Figure 6. This section was intended to prove two hypotheses through numerical analysis: (a) that the electrical shape factor, $S_{e}$ is analogous to thermal shape factor, $S_{t}$; and (b) at two contact surfaces, the shape factor, $S$ is independent of the shape, i.e. triangle, circle or square as long as the conduction geometries: length, $L$ and area, $A$, remain constant. The geometry shown in Figure 5 represents the flux tube called a Holm tube which was initially used by some researchers (Negus, Yovanovich, \& Beck, 1989; Salgon, Robbe-Valloire, Blouet, \& Bransier, 1997; Yovanovich, 1976) to study the effect of thermal contact resistance across a single medium contact spot. This concept was adopted in this study to investigate the effect of various shapes on the shape factor at a single contact spot, and make a comparison between the electric shape factor and the thermal shape factor using the numerical approach. For all shapes shown in Figure 6, the conduction geometries: length, $L$ and area, $A$ were maintained as constant.

\section{SHAPE FACTOR ANALOGY}

The results obtained from the electrical measurements were the potential difference, $V$, which was used to calculate the electrical resistance, $R_{e}$ based on the Ohm's law as follows.

$$
V=I R
$$

The shape factor for electrical conduction, $S_{e}$ obtained from the field experiment, is calculated based on the electrical resistivity shown in Eq. (2). 


$$
\begin{gathered}
R_{e}=\rho(L / A) \\
S_{e}=A / L=\rho / R_{e}
\end{gathered}
$$

where $\rho$ is the electrical resistivity of constantan $\left(5.00 \times 10^{-7} \Omega . \mathrm{m}\right)$.

Thermal conduction is analogous to electrical conduction, and the thermal shape factor, $S_{t}$ can be calculated based on the Fourier's law of conduction as follows in Eq. (4).

$$
S_{t}=A / L=Q /(k \cdot \Delta T)
$$

Shape factor is a dimensional quantity. Based on the same dimensions, the value of the shape factor for both electrical and thermal conduction is the same.

$$
S_{t} \equiv S_{e}
$$

Several known conduction shape factors for several geometries are widely available in the literature (Sunderland \& Johnson, 1964). Some of these, such as plane wall, hollow cylinders, hollow square passage and hollow cylinder in square bar, shown in Figure 7, were used in the numerical study for validation purposes.

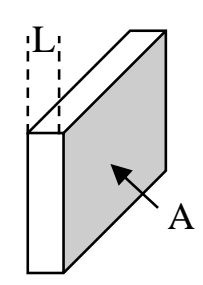

Plane wall

$S=A / L$

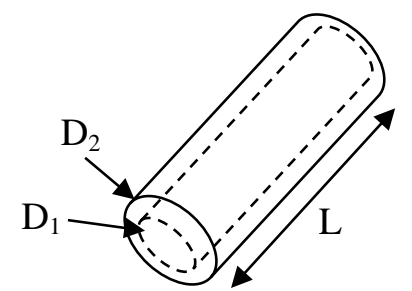

Hollow cylinder

$$
S=\frac{2 \pi L}{\ln \left(D_{2} / D_{1}\right)}
$$

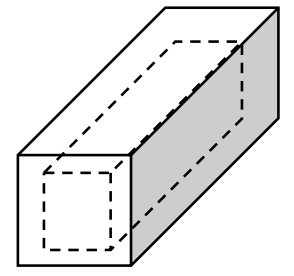

Hollow square passage

For $\mathrm{a} / \mathrm{b}>1.4$ :

$S=\frac{2 \pi L}{0.93 \ln (0.948 a / b)}$

For $\mathrm{a} / \mathrm{b}<1.41$ :

$S=\frac{2 \pi L}{0.785 \ln (a / b)}$

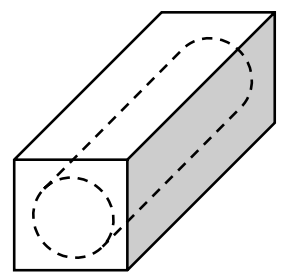

Hollow cylinder in square bar

$S=\frac{2 \pi L}{\ln (1.08 w / D)}$

Figure 7. Known conduction shape factors.

\section{RESULTS AND DISCUSSION}

The discussion of the results will focus on four main effects: the effect of bonding, the effect of geometrical shape, the effect of shape length, and the effect of fin design. The numerical results were validated by comparing the shape factor, $S$, from the experiments as shown in a later section. Prior to carrying out the numerical analysis, grid independence tests were performed to obtain appropriate mesh relevance. The grid independence test is shown in Figure 8 and as a result, a mesh relevance of 40 was found to be sufficient to perform the numerical tests. This mesh relevance in ANSYS is a method to refine the mesh size in which the range of mesh relevance is given from 0 ((high speed solution, coarser mesh, less accuracy) to 100 (slower speed solution, finer 
mesh, higher accuracy) as detailed in the ANSYS User Guide. In addition to mesh relevance, ANSYS also allows secondary refinement of the mesh by adjusting predefined options such as coarse, medium and fine modes. In this study, fine mode and a mesh relevance of 40 were selected.

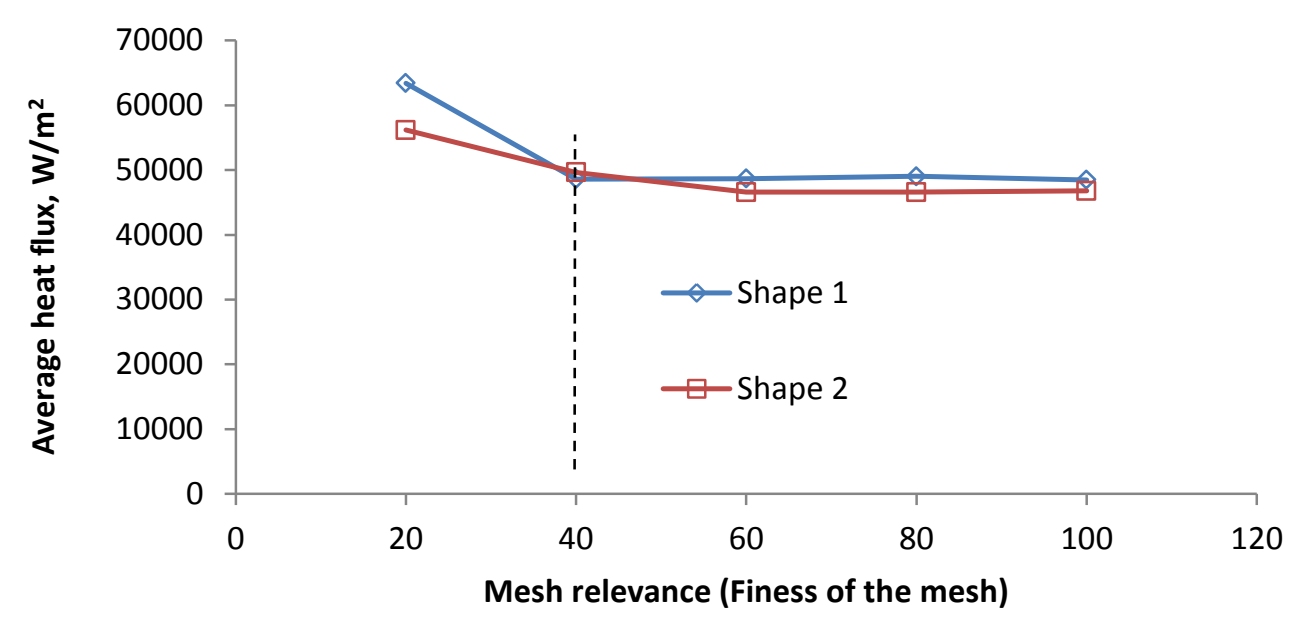

Figure 8. Grid independence test.

In the numerical analysis, a comparison between the electrical and the thermal shape factors was conducted using the analysis modules provided in the numerical software. For this comparison, simple geometries were used, such as round, square and triangular shapes. For the thermal model, the wall boundaries were set at $30^{\circ} \mathrm{C}$ and $50^{\circ} \mathrm{C}$, respectively, while for the electrical model, the boundaries were set at a current of $1 \mathrm{~A}$. In these two cases, the rest of the conditions were well insulated, and the ambient temperature was set at $22^{\circ} \mathrm{C}$. The numerical results of the shape factor in the electrical and thermal modules show very close agreement, with an approximately $1.0 \%$ deviation. This slight deviation is only due to round-off error. A summary of this result is shown in Figure 9. Based on Figure 9, of both the analysis modules, it can be concluded that both the thermal and electric module predicted the shape factor with a small error and thus the electric analysis module was sufficient to estimate the shape factor. Its prediction accuracy has been verified by experiment which will be discussed in following section.

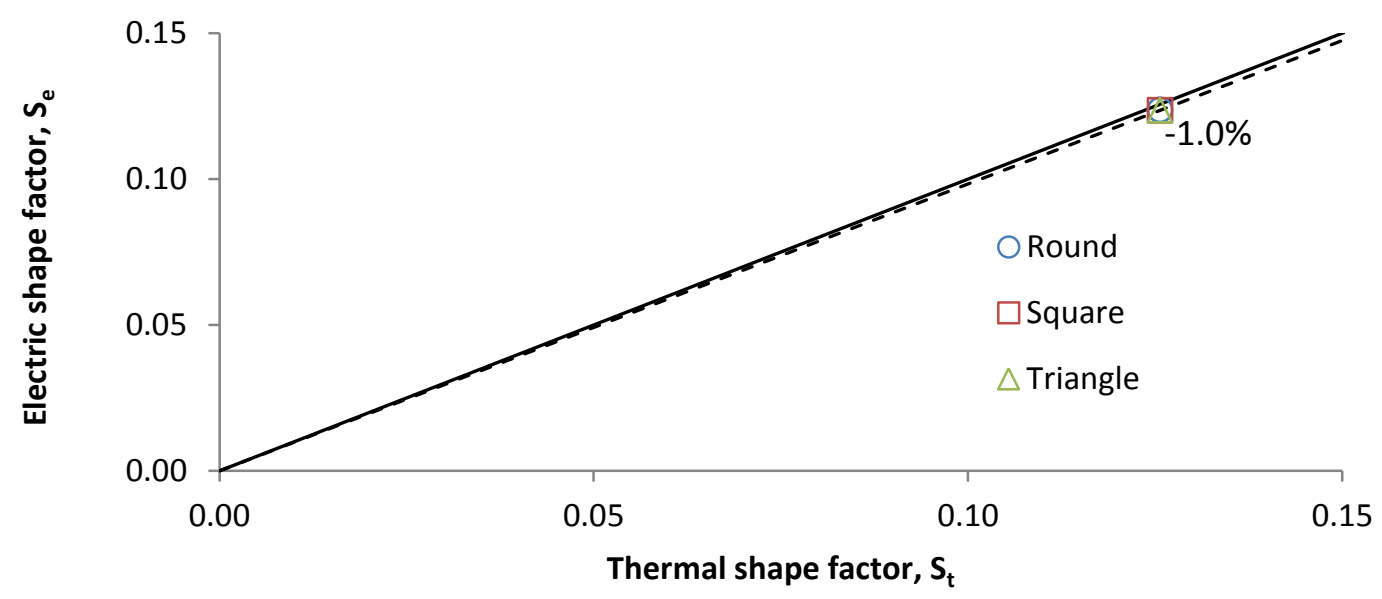

Figure 9. Shape factor comparison between thermal and electric module. 


\section{Effect of Bonding}

Prior to the field experiments, the bonding between the copper wires and the constantan foil was assessed. This was done to ensure that the resistance between the wires and the foil did not affect the actual reading of the fin (or foil) resistance. Three methods were adopted for this bonding: (a) using silver conductive adhesion (Madhusudana, 1996), (b) brazing using silver flux, and (c) brazing using cesium-aluminum flux. The results are shown in Figure 10. As shown in Figure 9, the bonding between the wires and the foil is most effective with brazing using cesium-aluminum flux which offered the least resistance, approximately $26 \%$ on average lower than the silver paint method. Silver paint had the highest resistance as it only provided adhesion between the wires and the foil.

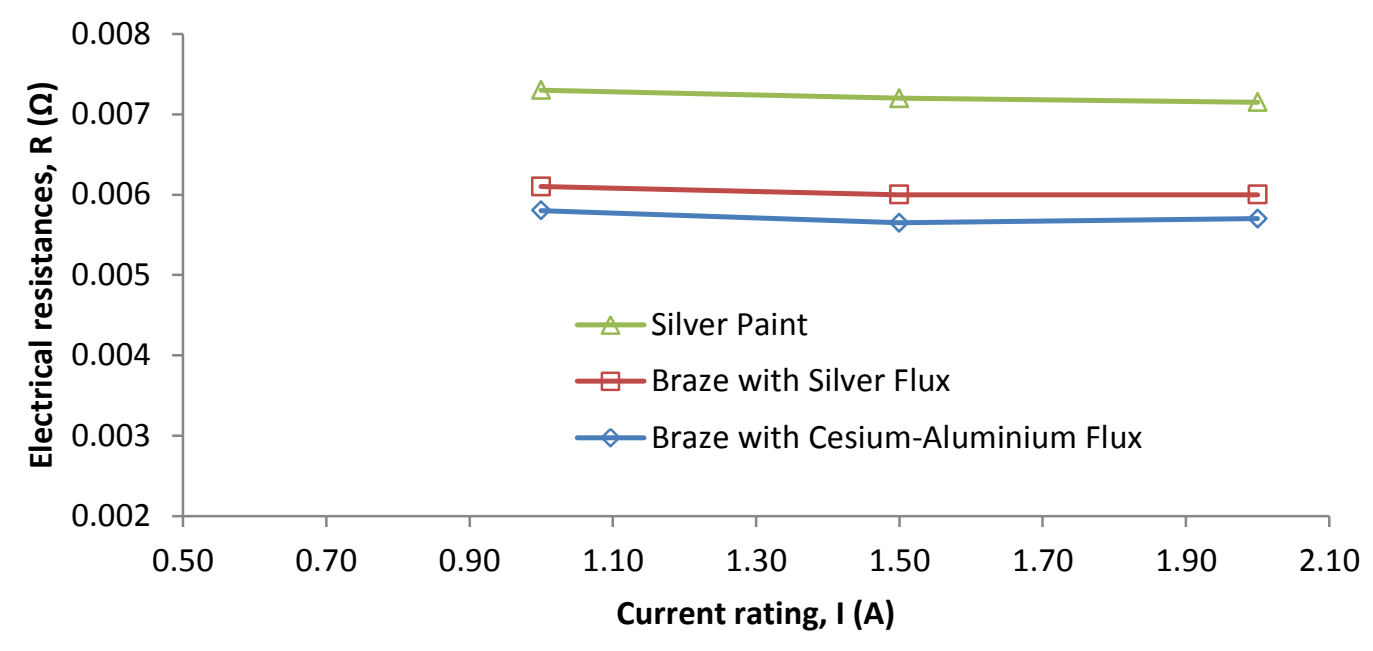

Figure 10. Effect of wires bonding on the foil with different methods.

\section{Effect of Shape}

The effect of different shapes serves as a validation between the numerical and experimental analyses. Several known shape factor formulae, such as those for plane wall, hollow cylinder and hollow square passage, shown in Figure 6, were used to compare the numerical results, which were based on the electric shape factor. In all the cases shown in Figure 11, the numerical results agreed well with the known formulae and there was almost exact agreement for plane wall and hollow cylinder. The deviation was only $0.16 \%$ and $1.15 \%$ for the hollow cylinder in a square bar and the hollow square passage, respectively. Actual experiments on the shape factor were conducted to further verify the numerical analysis. The numerical models for this investigation are shown in Figure 3 where Shape 1 is considered as the baseline while Shapes 2 and 3 are at different sizes, as aforementioned. The experiment followed the methodology explained earlier in Section 2.1. The results of this comparison are shown in Figure 12. 


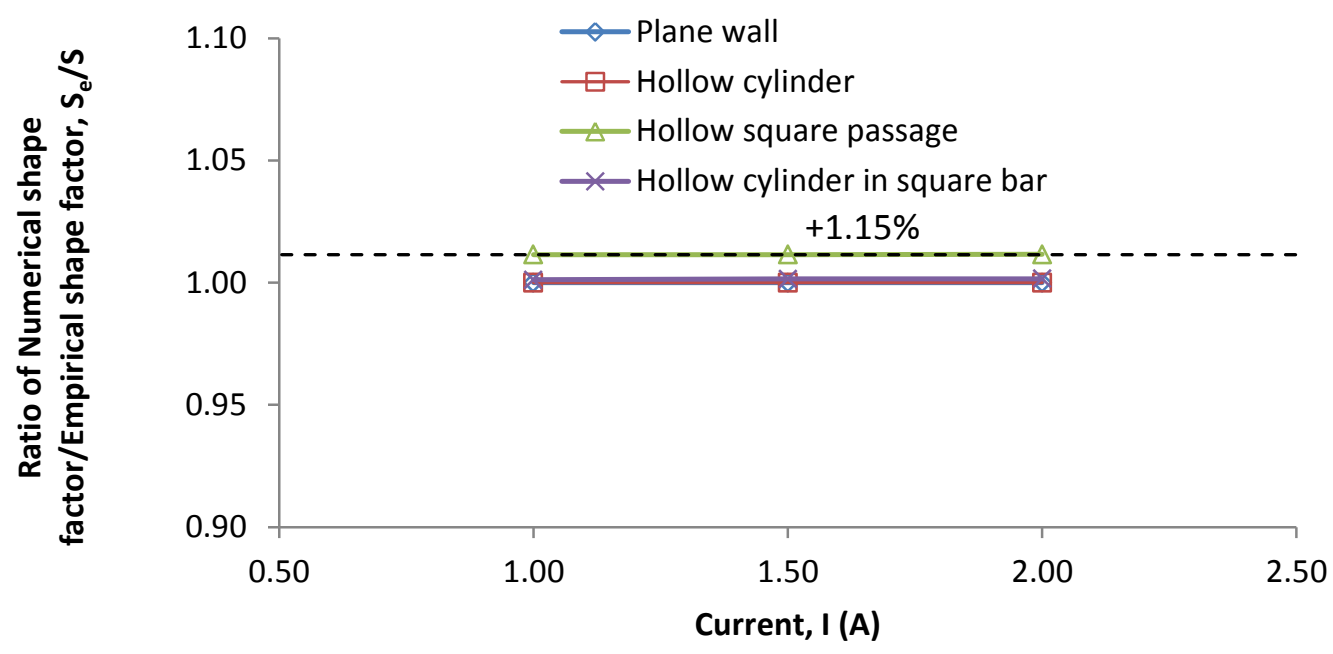

Figure 11. Comparison of shape factors between known equations and numerical result.

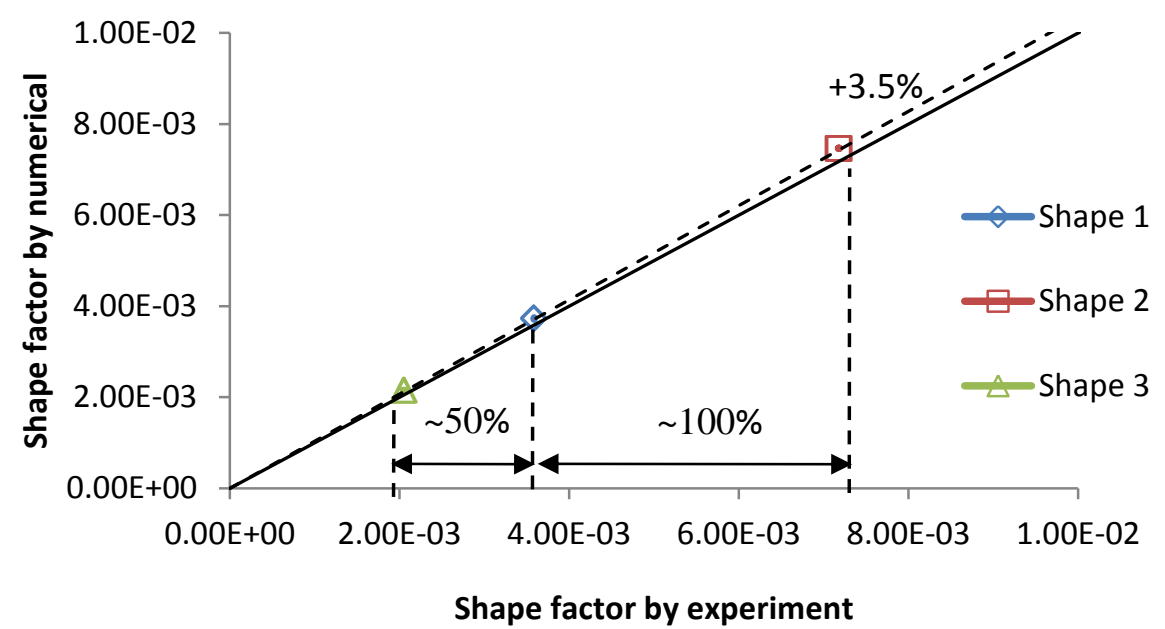

Figure 12. Comparison between numerical and experimental shape factor, $S$.

Based on Figure 12, the uncertainty between numerical and experimental results was about $+3.5 \%$ for all 3 shapes, where the numerical results are slightly higher than the experiments. This was due to minor rogue resistances that might occur in experiments. As the resistance is reciprocal to shape factor as shown in Equations (2) and (3), the shape factor of the experiment will be lower than the numerical result. The uncertainty of this result is thus still within an acceptable tolerance range.

\section{Effect of Conduction Length and Area}

The effects of shape conduction length and area are shown in Figure 12, as Shape 2 refers to a size increment of the original scale by a factor of 2 maintaining the geometric similarity, and Shape 3 refers to a smaller size which is half the original scale. The conduction length here refers to the row pitch, $P_{r}$ between tube collars from one row to another, as shown in Figure 2. From Figure 12, it can be seen that when the size was increased by a factor of 2 , the shape factor also doubled and when the size was reduced 
to half that of the original, the shape factor decreased proportionately by about $50 \%$. It can thus be concluded that the shape factor, $S$, based on Equations (3) and (4) is purely the influenced by the conduction path or the length across the foil.

\section{Effect of Waviness of Fin}

Since the numerical model was validated experimentally, it was decided to use it to investigate the characteristics of different fin designs, including wavy fin type. Figure 13 shows the vectors of flux lines across the constantan foil for Shapes 4 and 5 based on the wavy fin design, which has not been investigated before.

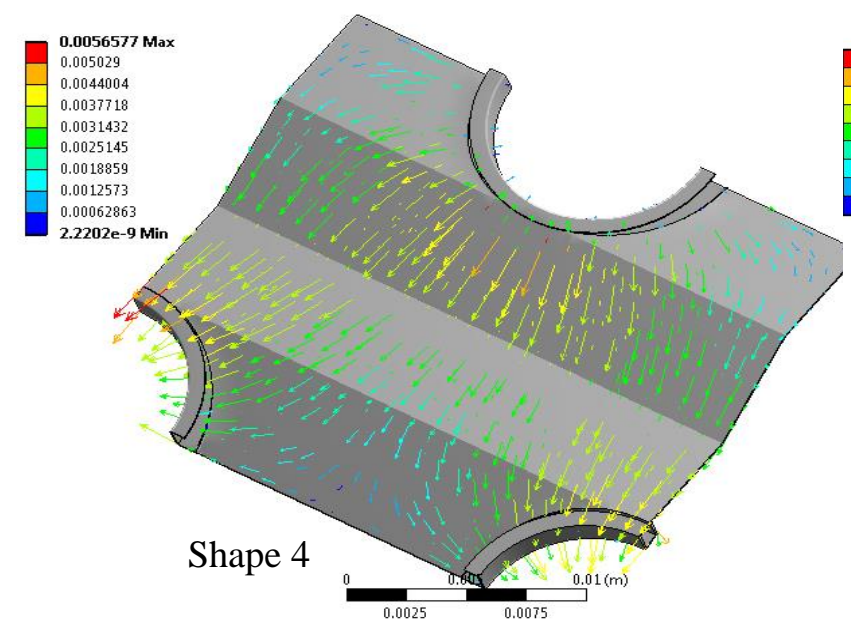

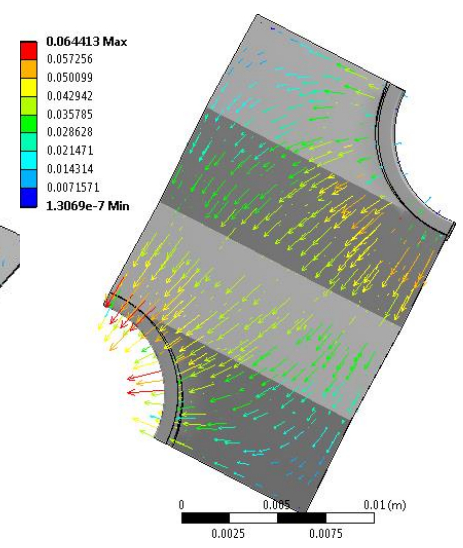

Shape 5

Figure 13. Vectors of electrical field across the constantan foil.

Figure 13 also clearly shows the path of the electrical field from one tube collar to another. Shape 5 is half of the symmetrical geometry of Shape 4, and it was expected to have half the shape factor of Shape 4. The results confirmed this argument, as shown in Figure14. The shape factor of the wavy fin (Shape 4) was then compared with the plain fin (Shape 1) and the results are shown in Figure 15. It can be seen that the wavy fin has a lower shape factor than plain fin by about 3.0\% due to the longer conduction length of the wavy fin.

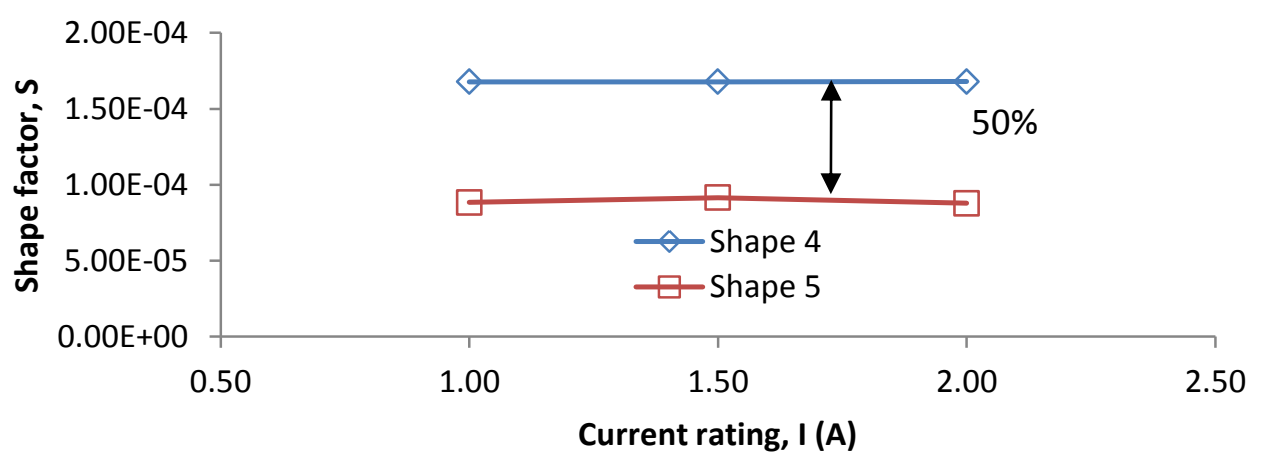

Figure 14. Shape factor, $S$, for Shape 4 and 5 at different current rating, $I$. 


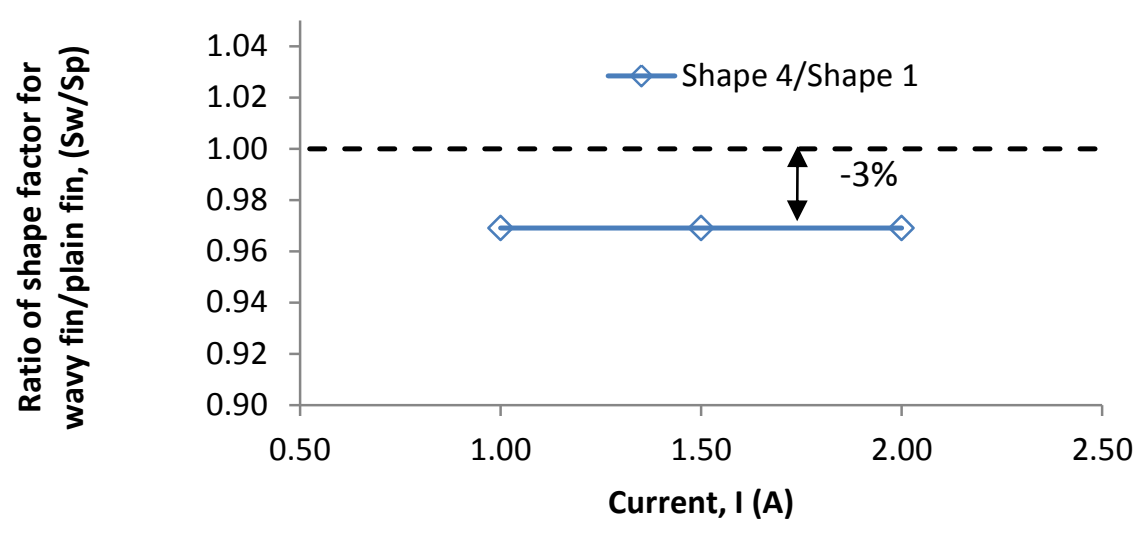

Figure 15. Ratio of shape factor for wavy fin/plain fin (Shape 4/Shape 1).

\section{CONCLUSIONS}

Based on the experimental and numerical results of the shape factor, the method to obtain the complex geometry of the wavy fin is established, which shows agreement within $+3.5 \%$ for different shapes and current ratings. The wavy fin design was simulated experimentally, and numerically based on the actual dimensions, and its shape factor was obtained. Other conclusions can be drawn from this investigation, such as that the efficiency of the bonding between copper wires and the test unit, constantan foil for electrical experiment, was demonstrated to be adequate with brazing using cesium-aluminum flux. The shape factor is a linear function of the conduction length, $L$ and area, $A$. For any two contacting surfaces, the shape factor does not vary with the shapes, as long as the conduction geometries, length and area, remain constant. The shape factor will be useful in future investigations of the contact resistance between fins and tubes which is part of the total thermal resistance of a fin-tube heat exchanger.

\section{ACKNOWLEDGMENTS}

The authors would like to acknowledge and extend their gratitude to the support given by O.Y.L. R\&D SDN BHD.

\section{REFERENCES}

Al- Doori, W. H. A. R. (2011). Enhancement of natural convection heat transfer from the rectangular fins by circular perforations. International Journal of Automotive and Mechanical Engineering, 4, 428-436.

Bart, G. C. J., \& Hanjalić, K. (2003). Estimation of shape factor for transient conduction. International Journal of Refrigeration, 26(3), 360-367.

Cengel, Y. A., Boles, M. A., \& Kanoglu, M. (2007). Thermodynamics: An engineering approach (si units) (Vol. 6). Singapore: McGraw-Hill New York.

Fyrillas, M. M. (2008). Heat conduction in a solid slab embedded with a pipe of general cross-section: Shape factor and shape optimization. International Journal of Engineering Science, 46(9), 907-916. 
Fyrillas, M. M. (2010). Shape factor and shape optimization for a periodic array of isothermal pipes. International Journal of Heat and Mass Transfer, 53(5-6), 982-989.

Incropera, F. P., \& De Witt, D. P. (2002). Introduction to heat transfer. New York: John Wiley \& Sons.

Ishak, M., Tahseen, T. A., \& Rahman, M. M. (2013). Experimental investigation on heat transfer and pressure drop characteristics of air flow over a staggered flat tube bank in crossflow. International Journal of Automotive and Mechanical Engineering, 7, 900-911.

Madhusudana, C. V. (1996). Thermal contact conductance. New York: Springer-Verlag.

Negus, K. J., Yovanovich, M. M., \& Beck, J. V. (1989). On the nondimensionalization of constriction resistance for semi-infinite heat flux tubes. Journal of Heat Transfer, 7(111), 804-807.

Salgon, J. J., Robbe-Valloire, F., Blouet, J., \& Bransier, J. (1997). A mechanical and geometrical approach to thermal contact resistance. International Journal of Heat and Mass Transfer, 40(5), 1121-1129.

Shrivastava, D., \& Roemer, R. (2003). A comparison between 2-d and 3-d conduction shape factors. Paper presented at the ASME 2003 Heat Transfer Summer Conference.

Shrivastava, D., \& Roemer, R. (2005). An analytical study of 'poisson conduction shape factors' for two thermally significant vessels in a finite, heated tissue. Physics in Medicine and Biology, 50(15), 3627.

Sunderland, J. E., \& Johnson, K. R. (1964). Shape factors for heat conduction through bodies with isothermal or convective boundary conditions. ASHRAE Trans, 70, 237-241.

Syam Sundar, L., \& Sharma, K. V. (2011). Laminar convective heat transfer and friction factor of al2o3 nanofluid in circular tube fitted with twisted tape inserts. International Journal of Automotive and Mechanical Engineering, 3, 265-278.

Teertstra, P. M., Yovanovich, M. M., \& Culham, J. R. (2005). Conduction shape factor models for three-dimensional enclosures. Journal of Thermophysics and Heat Transfer, 19(4), 527-532.

Yovanovich, M. M. (1973). A general expression for predicting conduction shape factors 11th aerospace sciences meeting (pp. 73-121): American Institute of Aeronautics and Astronautics.

Yovanovich, M. M. (1976). General expression for circular constriction resistances for arbitrary flux distributions. Progress in Astronautics and Aeronautics: Radiative Transfer and Thermal Control, 49, 381-396. 